\title{
The SYK model and random tensors: Gaussian universality
}

\section{T. Krajewski*}

Aix Marseille Univ, Université de Toulon, CNRS, CPT, Marseille, France

E-mail: thomas.krajewski@cpt.univ-mrs.fr

\section{Laudonio}

Université de Bordeaux, LaBRI, CNRS UMR 5800, Talence, France, EU

Department of Applied Mathematics, University of Waterloo, Waterloo, Ontario, Canada

E-mail: matteo.laudoniodu-bordeaux.fr

\section{R. Pascalie}

Université de Bordeaux, LaBRI, CNRS UMR 5800, Talence, France, EU

Mathematisches Institut der Westfalischen Wilhelms-Universitaat, Münster, Germany, EU

E-mail: romain.pascalie@u-bordeaux.fr

\section{A. Tanasa}

Université de Bordeaux, LaBRI, CNRS UMR 5800, Talence, France, EU

H. Hulubei Nat. Inst. Phys. Nucl. Engineering, Magurele, Romania, EU

IUF Paris, France, EU

E-mail: ntanasa@u-bordeaux.fr

The SYK model involves N Majorana fermions in 1+0 dimensions with quenched Gaussian disorder and proves to be exactly solvable in the large $\mathrm{N}$ limit at strong coupling. It has been initially proposed by Sachdev and Ye as a model of condensed matter and later gained some interest as a toy model of AdS/CFT correspondence, thanks to the work of Kitaev. On the other side, random tensors are generalisations of random matrices to objets that carry more than two indices. It turns out that the SYK model and random tensors involve a special class of Feynman graphs known as "melons". We will briefly review both constructions. Then, we will show how non Gaussian disorder can be reduced to a Gaussian one, treating the coupling as a random tensor, thanks to Gurau's Gaussian universality result.

Corfu Summer Institute 2019 "School and Workshops on Elementary Particle Physics and Gravity" (CORFU2019)

31 August - 25 September 2019

Corfù, Greece

\footnotetext{
* Speaker.
} 


\section{Introduction}

The Sachdev-Ye-Kitaev (SYK) model is a quantum mechanical model (equivalently, a quantum field theory in $1+0$ dimensions) involving $N$ Majorana fermions $\psi_{1}(t), \ldots, \psi_{N}(t)$ submitted to quenched disorder $J_{i_{1}, \ldots, i_{q}} \psi_{i_{1}}(t) \cdots \psi_{i_{q}}(t)$, with $J_{i_{1}, \ldots, i_{q}}$ a Gaussian random coupling. It originated in condensed matter theory, see [1] and [2], and was later proposed as a model of $\mathrm{AdS}_{2} / \mathrm{CFT}_{1}$ duality; in the large $N$ limit and at strong coupling, see [3], [4] and [5], or the recent reviews [6] and [7].

On the other side, random tensors are natural generalisations of random matrices $M_{i j} \rightarrow T_{i j k \ldots}$, with $\mathrm{U}(N)$ or $\mathrm{O}(N)$ invariant probability laws. Following the relation between random matrices and 2D quantum gravity, they have been introduced in the nineties as generating functions for random higher dimensional discrete geometries, defined as weighted triangulations,

$$
\int_{\text {rank } D \text { tensors }} d T \exp -S_{N}(T)=\sum_{\substack{D \text {-valent Feynman graphs } \\ \Leftrightarrow D \text {-dimensional triangulations }}} W(\text { triangulation }) .
$$

Progress in this direction has been slow until a suitable large $N$ limit has been found by Gurau [8], see also the monograph [9] for an in-depth account of random tensors.

The Feynman graph expansion of (1.1) involves a particular class of graphs termed "melons" [10] which are obtained by recursive insertions of dressed propagators on the edges. These are precisely the graphs that dominate the large $N$ behaviour of the SYK model (see [11] for a complete analysis), so that a reformulation of the SYK model in the framework of random tensors has been proposed by Witten [12]. We refer the reader to the reviews [13] and [14] for recent overviews.

In this talk, we present an other application of random tensors to the SYK model in order to address the question of averaging over a non Gaussian disorder. To do so, we treat the random coupling $J_{i_{1}, \ldots, i_{q}}$ as a random tensor. Thanks to Gaussian universality [15], it turns out that a non Gaussian disorder is equivalent to a Gaussian one with modified parameters. This is an adaptation to the SYK model of some former result by Bonzom, Gurau and Smerlak in the case of p-spin glasses [16]. We sketch here the general idea and refer to our original publication [17] for a detailed account.

\section{The Sachdev-Ye-Kitaev (SYK) model}

The basic degrees of freedom of the SYK model are quantum mechanical Majorana fermions $\psi_{1}(t), \ldots, \psi_{N}(t)$. In the canonical formalism, the fermion creation/annihilation are identical and obey the anti-commutation rule

$$
\left\{\psi_{i}, \psi_{j}\right\}=\delta_{i j}
$$

These fermion interact through an even degree $q$ random interaction, so that its hamiltonian reads

$$
H=\mathrm{i}^{q / 2} J_{i_{1} \ldots i_{q}} \psi_{i_{1}} \cdots \psi_{i_{q}},
$$

where $J_{i_{1} \ldots i_{q}} \in \mathbb{R}$ is a fully antisymmetric Gaussian random coupling with mean 0 and covariance

$$
\left\langle J_{i_{1} \ldots i_{q}} J_{j_{1} \ldots j_{q}}\right\rangle=\frac{\sigma^{2}}{N^{q-1}} \sum_{\text {permutations } \pi} \varepsilon(\pi) \delta_{i_{1} j_{\pi(1)}} \cdots \delta_{i_{q} j_{\pi(q)}} .
$$


The standard deviation $\sigma$ is a measure of the size of the disorder and the scaling in $N$ is devised so that there is a finite large $N$ limit, as we will shortly see. The occurence of a sum over permutations, weighted by the signature, arises from the antisymmetric nature of the coupling. This coupling represents some quenched disorder, which signifies that one has to average extensive/connected quantities. In statistical mechanics, this means that the disorder $J_{i_{1} \ldots i_{q}}$ is not a new degree of freedom but rather an external parameter over which we average. The system may be considered as made of many subsystems, each with its own value of $J_{i_{1} \ldots i_{q}}$, which motivates the averaging of extensive quantities like the free energy $F=-T \log Z$. In this context, $J_{i_{1} \ldots i_{q}}$ is a rank $q$ tensor and averaging turns it into a random tensor, as we will detail in the next section.

There is a generalisation to a model of fermions with flavours, [18]: fermions have an extra label $a$ and the hamiltonian reads

$$
H=\mathrm{i}^{q / 2} J_{i_{1,1} \ldots i_{1, q_{1}}, \ldots, i_{f, 1} \ldots i_{1, q_{f}}} \prod_{a=1, \ldots, f} \psi_{i_{a, 1}}^{a} \cdots \psi_{i_{a, q_{a}}^{a}}^{a}
$$

This model shares many of the features of original SYK model and encompasses a lot of generalisations proposed so far.

As a first step, it is convenient to adopt a path integral point of view, using the action

$$
S=\int d t\left\{\psi_{i} \partial_{t} \psi_{i}-\mathrm{i}^{q / 2} J_{i_{1} \ldots i_{q}} \psi_{i_{1}} \cdots \psi_{i_{q}}\right\}
$$

where $\psi_{i}$ Grassmann variables. Considering the SYK model as a 1+0-dimensional quantum field theory, the Feynman rules at fixed $J_{i_{1} \ldots i_{q}}$ are as follows.

- The free propagator is proportional to the signum,

$$
i-j \quad \rightarrow \quad G_{0}\left(t, t^{\prime}\right)=\left\langle T \psi_{i}(t) \psi_{j}\left(t^{\prime}\right)\right\rangle=\frac{\delta_{i j}}{2} \operatorname{sgn}\left(t-t^{\prime}\right) .
$$

- The interaction vertex is (for $q=4$ )

$$
i_{1}-\left.\right|_{i_{2}} ^{i_{4}} i_{3} \rightarrow J_{i_{1} \ldots i_{4}}
$$

Generalisations to arbitrary even values of $q$ are straightforward, only the case $q=2$ is peculiar and ressembles more a random mass matrix than a disordered interaction.

- Finally, there is a sum over internal indices $i \in\{1, \ldots, N\}$, in addition to the integrals over times.

Then, one computes the free energy or connected correlations functions as a sum over connected graphs and then average over disorder. Since $J$ is Gaussian, the average of a Feynman graph (which is a monomial in $\mathbf{J}$ ) reduces by wick's theorem to contractions of pairs using (2.3).

In the large $N$ limit, the combination of the scaling in (2.3) and the summation over internal indices leads to some drastic simplifications. Indeed, average over the Gaussian interaction selects 
pairs of vertices, removes them and reconnects the remaining edges. This leads to a maximal number of independent summations if these edges belong to different connected components (after removal of the pair of vertices). The graphs having the leading order contribution in $N$ are therefore those graphs such that, for any vertex, there is another vertex so that removing both vertices leads to $q$ (the valence of each vertex) connected components. These graphs are usually called "melons". A few examples of graphs are given in figure 1: the first two are melons while the third one is not and is therefore sub-leading.

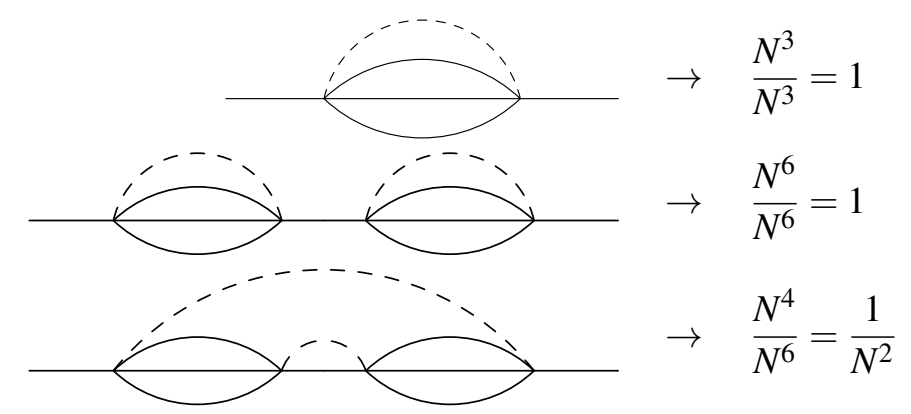

Figure 1: Some graphs for the two point function

Accordingly, leading order contributions to the 2-point function are obtained by repeated insertion on each line of a pari of vertices joined by $q-1$ dressed lines. This is conveniently encoded in the Schwinger-Dyson equation (see figure 2),

$$
G\left(t, t^{\prime}\right)=G_{0}\left(t, t^{\prime}\right)+\iint d u d v G_{0}(t, u) G^{q-1}(u, v) G\left(v, t^{\prime}\right) .
$$

Writing the integrals as convolution products, the Schwinger-Dyson equation are conveniently

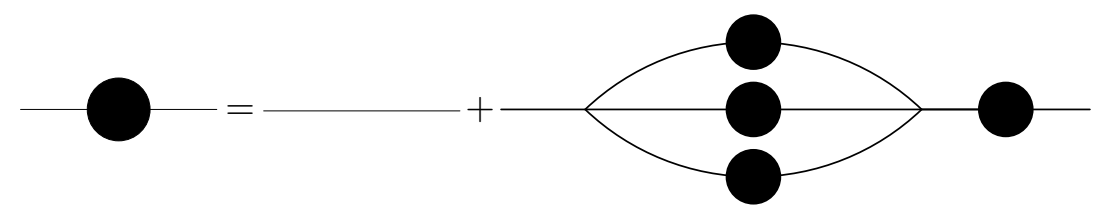

Figure 2: Schwinger-Dyson equation the two point function

abbreviated as

$$
G=G_{0}+G_{0} \star G^{q-1} \star G .
$$

Note that this equation sums all melons and is valid only at large $N$ (otherwise, the equations would be part of a larger system involving higher functions). If we further restrict to the large $J$ or equivalently large $t$ (infrared) regions, (2.11) admits a simple solution.

Indeed, at large $N$ in the IR, $N \gg J\left|t-t^{\prime}\right| \gg 1$, we may assume that $G \ll G_{0}$ and look for a conformal solution

$$
G_{*}\left(t, t^{\prime}\right) \propto \frac{\operatorname{sgn}\left(t-t^{\prime}\right)}{\left|t-t^{\prime}\right|^{2 \Delta}}
$$


This is a solution of the conformal approximation to (2.11)

$$
0=G_{0}\left(t, t^{\prime}\right)+\iint d u d v G_{0}(t, u) G^{q-1}(u, v) G\left(v, t^{\prime}\right) .
$$

provided $\Delta$ obeys

$$
0=0+2-(q-1) \times 2 \Delta-2 \Delta \quad \Rightarrow \quad \Delta=\frac{1}{q} .
$$

$\Delta$ is interpreted as the anomalous dimension of $\psi$ in the IR, while the canonical dimension of $\psi$ is 0 in the UV.

Similar results hold for the 4 point function: summing ladder graphs leads to an expression in terms of some hypergeometric function, we refer for instance to [4] and [5] for a complete account.

The previous large $N$ limit can be addressed in more conceptual way through the construction of an effective action in such a way that the large $N$ limit is nothing but a semi-classical one. This is done in several steps.

First, one computes the free energy, possibly in the presence of a source to generate connected correlations. In order to ease the average of $f=-T \log Z$, one uses the "replica trick". The latter is based on the simple identity

$$
\log (Z)=\lim _{n \rightarrow 0} \frac{Z^{n}-1}{n} .
$$

$Z^{n}$ is computed as the partition function of an ensemble of $n$ independent fermions $\psi_{i}(t) \rightarrow \psi_{i}^{r}(t)$ with $r=1, \ldots, n$, called replicas,

$$
Z^{n}=\int \prod_{r, i}\left[d \psi_{i}^{r}(t)\right] \exp -\sum_{r} \int d t\left\{\psi_{i}^{r} \partial_{t} \psi_{i}^{r}-\mathrm{i}^{q / 2} J_{i_{1} \ldots i_{q}} \psi_{i_{1}}^{r} \cdots \psi_{i_{q}}^{r}\right\}
$$

At a formal level, this procedure reduces the computation of the logarithm to a simple replication of the degrees of freedom. The quenched average over the disorder can therefore be explicitly performed,

$$
\langle\log Z\rangle_{J}=\lim _{n \rightarrow 0} \frac{1}{n}\left(\frac{\int d J_{i_{1} \ldots i_{q}} Z^{n} \exp -\frac{N^{q-1}}{2 q ! \sigma^{2}}\left(J_{i_{1} \ldots i_{q}} J_{i_{1} \ldots i_{q}}\right)^{2}}{\int d J_{i_{1} \ldots i_{q}} Z^{n} \exp -\frac{N^{q-1}}{2 q ! \sigma^{2}}\left(J_{i_{1} \ldots i_{q}} J_{i_{1} \ldots i_{q}}\right.}-1\right)
$$

To simplify this expression, one introduces composite degrees of freedom, bi-local in time

$$
G^{r r^{\prime}}\left(t, t^{\prime}\right)=\frac{1}{N} \sum_{i} \psi_{i}^{r}(t) \psi_{i}^{r^{\prime}}\left(t^{\prime}\right)
$$

with $r$ and $r^{\prime}$ replicas indices. The previous relation is enforced in the path integral using a Lagrange multiplier $\Sigma^{r r^{\prime}}\left(t, t^{\prime}\right)$. Then the integrals over $J$ and $\psi$ are Gaussian and can be performed, so that we are left with a functional integral over $G$ and $\Sigma$. Assuming replica diagonal solutions (see [21] for a discussion of non diagonal ones), $G^{r r^{\prime}}\left(t, t^{\prime}\right)=G\left(t, t^{\prime}\right) \delta^{r r^{\prime}}, \Sigma^{r r^{\prime}}\left(t, t^{\prime}\right)=\Sigma\left(t, t^{\prime}\right) \delta^{r r^{\prime}}$, we are left with

$$
\langle\log Z\rangle_{J}=\int[D G][D \Sigma] \exp N S_{\mathrm{eff}}[G, \Sigma]
$$


with the effective action

$$
S_{\mathrm{eff}}[G, \Sigma]=\frac{1}{2} \log \operatorname{det}(\partial-\Sigma)_{\star}+\frac{1}{2} \int d t d t^{\prime}\left\{\Sigma\left(t, t^{\prime}\right) G\left(t, t^{\prime}\right)+J G^{q}\left(t, t^{\prime}\right)\right\}
$$

Note that by construction $G$ and $\Sigma$ are $\mathrm{O}(N)$ scalars, so that $N$ only appears as a pre-factor in the exponential. Therefore, the large $N$ limits amounts to the saddle point approximation for stationary points $(G, \Sigma)$ of of the effective action. These equations are

$$
\begin{aligned}
G\left(t, t^{\prime}\right) & =\left[\delta\left(t-t^{\prime}\right) \partial_{t}-\Sigma\left(t, t^{\prime}\right)\right]_{\star}^{-1} \\
\Sigma\left(t, t^{\prime}\right) & =q \sigma^{2}\left[G\left(t, t^{\prime}\right)\right]^{q-1}
\end{aligned}
$$

Eliminating $\Sigma\left(t, t^{\prime}\right)$ with $G_{0}\left(t, t^{\prime}\right)=\left[\delta\left(t-t^{\prime}\right) \partial_{t}\right]_{\star}^{-1}$ the free propagator leads to

$$
G=G_{0}+G_{0} \star G^{q-1} \star G
$$

which is nothing but the Schwinger-Dyson equation (2.11).

I need to find out the right numerical factor in front of $G^{q}$, which I simply write $J$ and factor $1 / 2$.

In the IR, the effective action (2.22) is invariant under reprarametrisation $t \rightarrow f(t)$ with $\Delta=\frac{1}{q}$, provided

$$
\begin{aligned}
\psi(t) & \rightarrow\left|\frac{d f}{d t}\right|^{\Delta} \psi(f(t)) \\
G\left(t, t^{\prime}\right) & \rightarrow\left|\frac{d f}{d t}\right|^{\Delta}\left|\frac{d f}{d t^{\prime}}\right|^{\Delta} G\left(f(t), f\left(t^{\prime}\right)\right) \\
\Sigma\left(t, t^{\prime}\right) & \rightarrow\left|\frac{d f}{d t}\right|^{1-\Delta}\left|\frac{d f}{d t^{\prime}}\right|^{1-\Delta} \Sigma\left(f(t), f\left(t^{\prime}\right)\right)
\end{aligned}
$$

Note that the saddle point solution

$$
G\left(t, t^{\prime}\right) \propto \frac{\operatorname{sgn}\left(t-t^{\prime}\right)}{\left|t-t^{\prime}\right|^{2 \Delta}}
$$

is only invariant under the $\mathrm{SL}_{2}(\mathbb{R})$ transformations

$$
t \rightarrow f(t)=\frac{a t+b}{c t+d}
$$

with $a, c, d, d$ real numbers such that $a d-b c=1$. Reparametrisation invariance is therefore a spontaneously broken approximate symmetry, analogous to the chiral symmetry in QCD. It leads to pseudo-Goldstone modes that are described by the Schwartzian derivative (see [19] and [20] ).

In the remainder of the paper, we are interested in the extension of the previous formalism to averaging over non Gaussian disorder by promoting the coupling $J_{i_{1}, \ldots, i_{q}}$ to a rank $q$ random tensor. 


\section{A short introduction to random tensors}

Rank $q$ random tensors are arrays of $N^{q}$ numbers $T_{i_{1}, \ldots, i_{q}}$ submitted to given probability laws. The tensors may be real or complex and we do not impose any specific symmetries under permutations of the indices but assume $\mathrm{U}(N)$ or $\mathrm{O}(N)$ invariant probability laws. In the general case, we average over random tensors using

$$
\langle\cdots\rangle=\frac{\int d T \cdots \exp -S_{N}(T)}{\int d T \exp -S_{N}(T)} .
$$

The action usually starts with a quadratic terms and is perturbed by some higher degrees invariant interactions. For a real non symmetric tensor, it takes the form

$$
S_{N}(T)=\frac{1}{2} N^{q-1} T^{2}+V_{N}(T),
$$

where $T^{2}$ is a shorthand for the contraction $T^{2}=T_{i_{1} \ldots i_{q}} T_{i_{1} \ldots i_{q}}$.

We impose an invariance under $\mathrm{O}^{q}(N)=\mathrm{O}(N) \times \cdots \times \mathrm{O}(N)$ transformations

$$
T_{i_{1} \cdots i_{q}} \rightarrow T_{i_{1} \cdots i_{q}}^{\prime}=O_{i_{1} j_{1}}^{(1)} \cdots O_{i_{q} j_{q}}^{(q)} T_{j_{1} \cdots j_{q}} .
$$

Note that each factor of the group acts independently on the different indices, this is possible only because we do not impose any permutational symmetry between the indices. If the tensors were assumed to be fully antisymmetric, then the symmetry group would be reduced to $\mathrm{O}(N)$ because all the indices have to be treated in the same way.

For non symmetric tensors, all algebraic invariants are constructed using edge coloured $q$ valent graphs. This means that each vertex has valence $q$ (i.e. is attached to $q$ edges) and that each edge in the graph may be assigned a number in $\{1,2, \ldots, q\}$ in such a way that at each vertex all the edges attached to that vertex carry different numbers. The invariants are constructed by assigning tensors to the vertices and contracting the indices pairwise along the edges, the position in the tensor being given by the label. Denoting by $\Gamma$ such a graph, we write the invariant as $(T \cdots T)_{\Gamma}$ (as many tensors as there are vertices in the graphs), which stands for products of tensors contracted along the graph. We give a few examples for a rank 3 tensor in figure 3. In this figure, the first graph is a dipole and represents the kinetic term. The next is a quartic melonic interaction and the last one a tetrahedral interaction.

For non symmetric complex tensors, we treat $T$ and $\bar{T}$ as independent variables. The invariance group is now $\mathrm{U}^{q}(N)=\mathrm{U}(N) \times \cdots \times \mathrm{U}(N)$. The invariants for complex tensors involve bipartite coloured graphs of valence $q$. A graph are bipartite if we can colour the vertices in black or white, in such a way that edges only relate vertices of different colours. The constraints on the numbering of the edges remains unchanged. Then, the invariant is build by assigning $T$ to white vertices and $\bar{T}$ to black ones, and contracting along the edges. Let us also note the existence of the "multiorientable" tensor, where two indices transform withU $(N)$ and the remaining one with $\mathrm{O}(N)$, [22]. Finally, if the tensor have extra permutation symmetries, one should work with equivalence classes of graphs respecting these symmetries.

Of course, random tensors reduce to random matrices for $q=2$. In this case, graphs with vertices of valence 2 are disjoint union of cycles, each cycle with $v$ vertices yielding the invariant $\operatorname{Tr} M^{v}$. 


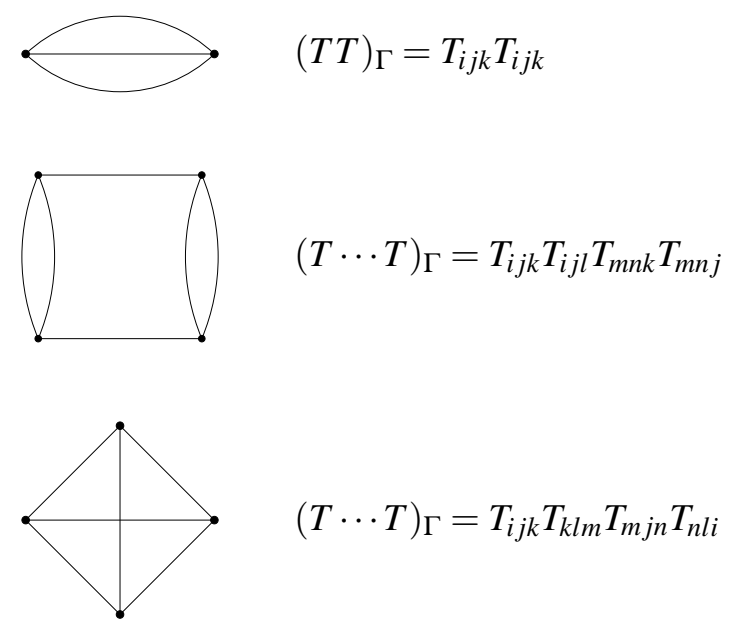

Figure 3: Some graphs invariants for a rank three real tensor

Then the potential in (3.2) is expanded over graph invariants as

$$
V_{N}(T)=\sum_{\Gamma} \frac{N^{\delta_{\Gamma}} \lambda_{\Gamma}}{\mathrm{S}_{\Gamma}}(T \cdots T)_{\Gamma}
$$

$\lambda_{\Gamma}$ is a coupling constant, $\mathrm{S}_{\Gamma}$ a combinatorial factor and $N^{\delta_{\Gamma}}$ a power of $N$ suitably chosen in such a way that there is a non trivial large $N$ limit. The existence of a non trivial large $N$ limit is a delicate question which can be formulated as follows. Determine the scaling of the interactions $N^{\delta_{\Gamma}}$ in such a way that, for a large class of observables $O(T)$, one has a finite nontrivial limit, possibly after rescaling, of the following expectation values

$$
\lim _{N \rightarrow \infty} \frac{1}{N^{\delta(O)}}\langle O(T)\rangle=\lim _{N \rightarrow \infty} \frac{1}{N^{\delta(O)}} \frac{\int d T O(T) \exp -S_{N}(T)}{\int d T \exp -S_{N}(T)} .
$$

For random matrices, this is easy : it suffices to take $\delta=1$ for every cycle so that $\frac{1}{N}\left\langle\operatorname{tr} M^{p}\right\rangle$ admits a finite non trivial limit.

The case of random tensor was not solved before a breakthrough by Gurau [8] for non symmetric tensors complex tensors. In this case, for a rank $q$ tensor, the scaling exponent is simply $\delta_{\Gamma}=q-c_{\Gamma}$, with $c_{\Gamma}$ the number of connected components. This ensures that every observable, build itself from a graph, has a finite limit when $N \rightarrow \infty$, after rescaling by a suitable power of $N$. In this case, only melonic graph observables have non zero limit and only depend on the melonic interactions in the potential. Moreover, the expectation can be computed with a modified Gaussian distribution, (see [15]), a fact that turns out to be crucial in our presentation of Gaussian universality for SYK models.

Many other tensor models have be shown to admit a large $N$ limit. Especially interesting for us are the real non symmetric rank 3 tensors with tetrahedral interaction have been studied by Carrozza and Tanasa [23]. in this case, the scaling is the usual one for the quartic melon $\delta_{\square}=2$ while the tetrahedron is enhanced, $\delta_{4}=5 / 2$ [23]. Let us also note that large $N$ limits may also 
be obtained for tensors with permutational symmetries [24]. This limit also exist with different scalings for the interactions [25], and lead to an extension of Gaussian universality.

These limits have been obtained using combinatorial techniques based on the Feynman graphs that appear in the expansion of (3.1) in powers of the couplings $\lambda-\Gamma$. These graphs have been shown to be melons [10]. Since these are precisely the graphs that occur in the SYK model after disorder averaging, tensor models have been used by Witten to reformulate the SYK model without disorder [12]. In this case, the fundamental degrees of freedom of the SYK model become tensors $\psi_{i j k \ldots}(t)$ instead of $\psi_{i}(t)$. Then, it is the tensorial structure of the index contractions that reproduces the large $N$ limit originally provided by the disorder.

In this talk, we are studying a different use of random tensors in the context of the SYK model: we propose to treat the coupling $J_{i_{1}, \ldots, i_{q}}$ as a random tensor and investigate the effects of interactions.

\section{Gaussian universality fro random tensors and the SYK model}

Turning back to the SYK model, let us focus on the disorder average using a non Gaussian weight,

$$
\langle\ldots\rangle_{J}=\frac{\int d J \ldots \exp -\left\{\frac{N^{q-1}}{2 \sigma^{2}} J^{2}+V_{N}(J)\right\}}{\int d J \exp -\left\{\frac{N^{q-1}}{2 \sigma^{2}} J^{2}+V_{N}(J)\right\}}
$$

where the potential $V_{N}(J)$ perturbation is a perturbation of the Gaussian distribution. Working with replicas, the average of the interaction term over the random tensor $J_{i_{1} \ldots i_{q}}$ reads

$$
\left\langle\exp \left\{J_{i_{1} \ldots i_{q}} \sum_{r} \int d t \psi_{i_{1}}^{r}(t) \cdots \psi_{i_{q}}^{r}(t)\right\}\right\rangle_{J}=\exp \left\{\frac{N^{q-1}}{2 \sigma^{2}} K^{2}-V_{N}^{\prime}(K)\right\}
$$

with

$$
K_{i_{1} \ldots i_{q}}=\frac{\sigma^{2}}{N^{q-1}} \sum_{r} \int d t \psi_{i_{1}}^{r}(t) \cdots \psi_{i_{q}}^{r}(t)
$$

and the normalised background effective potential $V_{N}^{\prime}(K)$ for $K$

$$
V_{N}^{\prime}(K)=-\log \int d J \exp -\left\{\frac{N^{q-1}}{2 \sigma^{2}} J^{2}+V_{N}(K+J)\right\}
$$

computed after shift of integration $J \rightarrow J+K$. Obviously, when $V_{N}=0$ we recover the Gaussian case. In the case of a invariant perturbation, both $V_{N}$ and $V_{N}^{\prime}$ can be expanded over graphs,

$$
V_{N}(T)=\sum_{\Gamma} \frac{N^{\delta_{\Gamma}} \lambda_{\Gamma}}{\mathrm{S}_{\Gamma}}(T \cdots T)_{\Gamma}, \quad V_{N}^{\prime}\left(T^{\prime}\right)=\sum_{\Gamma} \frac{N^{\delta_{\Gamma}} \lambda_{\Gamma}^{\prime}}{\mathrm{S}_{\Gamma}}(T \cdots T)_{\Gamma} .
$$

Thus, the couplings $\lambda_{\Gamma}^{\prime}$ can be computed as functions of $\lambda_{\Gamma}$ using (4.4). Then, the existence of a large $N$ limit can be formulated as finding suitable exponents $\delta_{\Gamma}$ such that $\lim _{N \rightarrow+\infty} \lambda_{\Gamma}^{\prime}\left(\lambda_{\Gamma}\right)$ exists. 
Let us now express the correction to the effective action $S_{\text {eff }}(G, \Sigma)$ (see equation (2.22)) due to the non Gaussian terms. As in the first section, we introduce the bi-local field

$$
G^{r r^{\prime}}\left(t, t^{\prime}\right)=\frac{1}{N} \sum_{i} \psi_{i}^{r}(t) \psi_{i}^{r^{\prime}}\left(t^{\prime}\right)
$$

and perform the Gaussian integration over $\psi_{i}^{r}(t)$. Under the assumption of a diagonal solution that does not break replica symmetry breaking, the new effective action reads

$$
S_{\text {eff }}^{\prime}(G, \Sigma)=S_{\text {eff }}(G, \Sigma)+\sum_{\Gamma} \frac{N^{\delta_{\Gamma}-1+e_{\Gamma}-(q-1) v_{\Gamma}} \lambda_{\Gamma}^{\prime}}{\mathrm{S}_{\Gamma}} \int \prod_{\substack{v \\ \text { vertices }}} d t_{v} \prod_{\substack{e=v^{\prime} \\ \text { edges }}} G\left(t_{v}, t_{v^{\prime}}\right),
$$

with $e_{\Gamma}$ the number of edges of $\Gamma$ and $v_{\Gamma}$ its number of vertices. This scaling in $N$ is understood as follows : each vertex yields a tensor $K$ and thus a power $N^{-(q-1) V_{\Gamma}}$ (see (4.3)) while each edge comes with a factor of $N$ (because of (4.6)); We further factorised a power of $N$ in front of the effective action. Since all graphs are $q$-valent, one has $2 e_{\Gamma}=q v_{\Gamma}$, so that the effective action is equivalently expressed as

$$
S_{\text {eff }}^{\prime}(G, \Sigma)=S_{\text {eff }}(G, \Sigma)+\sum_{\Gamma} \frac{N^{\delta_{\Gamma}-1-v_{\Gamma}(q / 2-1)} \lambda_{\Gamma}^{\prime}}{S_{\Gamma}} \int \prod_{\substack{v \\ \text { vertices }}} d t_{v} \prod_{\substack{e=v^{\prime} \\ \text { edges }}} G\left(t_{v}, t_{v^{\prime}}\right) .
$$

Note that if the perturbation $V$ is itself Gaussian, the only graph that contribute is the dipole (2 vertices related by $q$ edges) with $\delta \diamond=q-1$, so it is independent of $N$ as expected. It is also worthwhile to emphasise that the correction is invariant under the reparametrisation

$$
t \rightarrow f(t), \quad G\left(t, t^{\prime}\right) \rightarrow\left|\frac{d f}{d t}\right|^{\Delta}\left|\frac{d f}{d t^{\prime}}\right|^{\Delta} G\left(f(t), f\left(t^{\prime}\right)\right)
$$

with $\Delta=\frac{1}{q}$ since all vertices are $q$-valent. Therefore, all the reparametrisation breaking terms are included in the Gaussian contribution.

The large $N$ limit of the SYK model is always of a Gaussian nature if for all graphs $\delta_{\Gamma} \leq$ $1+v_{\Gamma}(q / 2-1)$, with equality only for the dipole graph, a property known as Gaussian universality. Note that in the special case $q=2$, the tensor model is just a random matrix with $\delta_{\Gamma}=1$ for single trace interactions and all cycles have finite non zero limits so that Gaussian universality does not hold.

Gaussian universality holds in the case of a complex non symmetric tensor [15].In our context, it corresponds to a modified version of the SYK model, the coloured complex SYK model with Hamiltonian

$$
H=\mathrm{i}^{\frac{q}{2}} \sum_{i_{1}, \ldots, i_{q}} \bar{J}_{i_{1}, \ldots, i_{q}} \psi_{i_{1}}^{1} \cdots \psi_{i_{q}}^{q}+\mathrm{i}^{\frac{q}{2}} \sum_{i_{1}, \ldots, i_{q}} J_{i_{1}, \ldots, i_{q}} \bar{\psi}_{i_{1}}^{1} \cdots \bar{\psi}_{i_{q}}^{q}
$$

The couplings $J_{i_{1}, \ldots, i_{q}}$ define a complex non symmetric tensor and the scaling of each perturbation is the canonical one, $\delta_{\Gamma}=q-c_{\Gamma}$, with $c_{\Gamma}$ the number of connected components. For instance, with a quartic "melonic" interaction,

$$
\longrightarrow \lambda N^{3} J_{i_{1} i_{2} i_{3} k} \bar{J}_{i_{1} i_{2} i_{3} l} \bar{J}_{j_{1} j_{2} j_{3} k} J_{j_{1} j_{2} j_{3} l}
$$




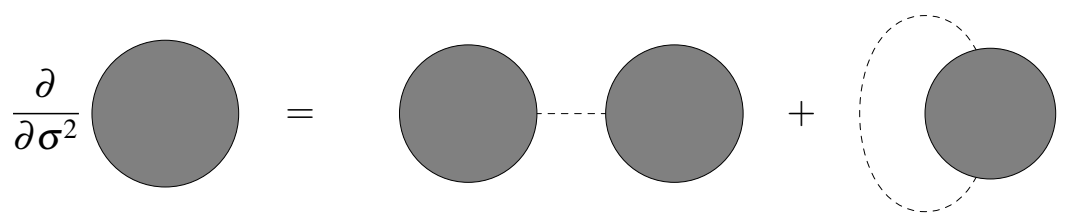

Figure 4: A Polchinski like equation for the effective potential $V_{N}^{\prime}$

Following [26], the existence of this large $N$ limit can be established using a variant of the celebrated Polchinski equation [27]. Indeed, the effective action for a complex non symmetric rank $q$ tensor is

$$
V_{N}^{\prime}\left(T^{\prime}, \bar{T}^{\prime}\right)=-\log \int d T d \bar{T} \exp -\left\{\frac{N^{q-1}}{2 \sigma^{2}} T \bar{T}+V_{N}\left(T^{\prime}+T, \bar{T}^{\prime}+\bar{T}\right)\right\} .
$$

It obeys a Polchinski-like equation, see figure 4

$$
\frac{\partial V_{N}^{\prime}}{\partial \sigma^{2}}=\frac{1}{N^{q-1}} \sum_{1 \leq i_{1}, \ldots, i_{q} \leq N}\left(\frac{\partial^{2} V_{N}^{\prime}}{\partial T_{i_{1}, \ldots, i_{q}} \partial \bar{T}_{i_{1}, \ldots, i_{q}}}-\frac{\partial V_{N}^{\prime}}{\partial T_{i_{1}, \ldots, i_{q}}} \frac{\partial V_{N}^{\prime}}{\partial \bar{T}_{i_{1}, \ldots, i_{q}}}\right)
$$

The various operations on the RHS of (4.14) are interpreted in terms of graphs as follows.

- Derivation with respect to $T$ and $\bar{T}$ removes a pair of vertices.

- Corresponding free lines are reattached by index contraction.

Because the scaling is $\delta_{\Gamma}=q-c_{\Gamma}$, the leading contributions arise when creating a maximal number of connected components,

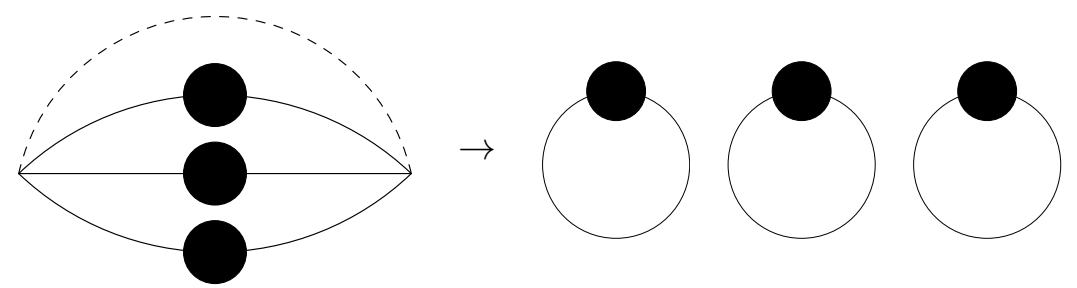

Then, a large $N$ limit exists so that the couplings $\lambda_{\Gamma}^{\prime}$ remain finite and only depend on the initial couplings $\lambda_{\Gamma}$ of melonic graphs. Moreover, since $\delta_{\Gamma}=q-c_{\Gamma}$ with $c_{\Gamma} \geq 1$, the exponent in (4.8) obeys $\delta_{\Gamma}-1-v_{\Gamma}(q / 2-1) \leq 0$ with equality only for the dipole. Therefore, the effective potential $V_{N}^{\prime}$ is dominated by the contribution to the dipole graph, which is equivalent to a modified Gaussian covariance (see [16]),

$$
\left(\sigma^{\prime}\right)^{2}=\frac{-1+\sqrt{1+4 \lambda \sigma^{4}}}{2 \lambda \sigma^{2}}
$$

The sub-leading order is a non Gaussian correction to the effective action given by

$$
\propto \frac{1}{N^{2}} \int d t_{1} d t_{2} d t_{3} d t_{4} G^{3}\left(t_{1}, t_{2}\right) G\left(t_{1}, t_{3}\right) G\left(t_{2}, t_{4}\right) G^{3}\left(t_{3}, t_{4}\right)
$$




\section{Conclusion and outlook}

In this talk, we have presented an other application of random tensors to the SYK model, treating the random coupling $J_{i_{1} \ldots i_{q}}$ as a random rank $q$ tensor. Provided a suitable large $N$ limits exists, Gaussian universality shows that the non Gaussian disorder can be reduced to a Gaussian one, albeit with a modified covariance. This is a salutary result since the structure of the SYK model at large $N$ heavily relies on Gaussian averages.

Strictly speaking, this result has been rigorously established only for the complex coloured SYK model, based on complex non symmetric tensors. However, this may also hold for other invariant models, for instance based on real antisymmetric tensors [24] or generalised melonic interactions [25]. A more radical modification could involve tensors with independent entries or the SYK models with lattice structure and/or global symmetries proposed in [28].

\section{References}

[1] S. Sachdev and J. Ye. Gapless spin fluid ground state in a random, quantum Heisenberg magnet. Phys.Rev.Lett., 70:3339, 1993.

[2] O. Parcollet and A. Georges. Non-Fermi-liquid regime of a dopped Mott insulator. Phys. Rev. B, 59:5341-5360, 1999.

[3] A. Kitaev. A simple model of quantum holography. KITP Program: Entanglement in Strongly-Correlated Quantum Matter, 2015.

[4] J. Maldacena and D. Stanford. Comments on the Sachdev-Ye-Kitaev model. Physical Review D, 94, 2016.

[5] J. Polchinski and V. Rosenhaus. The spectrum in the Sachdev-Ye-Kitaev model. Journal of High Energy Physics, 2016. arxiv:1601.06768.

[6] V. Rosenhaus. An introduction to the SYK model. arXiv:1807.03334 [hep-th], 2018.

[7] D. A. Trunin, Pedagogical introduction to SYK model and 2D Dilaton Gravity arXiv:2002.12187 [hep-th]

[8] R. Gurau. The complete 1/N expansion of a SYK-like tensor model. Nucl. Phys., B916:386-401, 2017.

[9] R. Gurau. Random Tensor Models. Oxford University Press, 2017.

[10] V. Bonzom, R. Gurau, A. Riello and V. Rivasseau, Critical behavior of colored tensor models in the large N limit Nucl. Phys. B 853 (2011), 174-195

[11] V. Bonzom, V. Nador, and A. Tanasa. Diagrammatic proof of the large $N$ melonic dominance in the SYK model. 2018.

[12] E. Witten, “An SYK-Like Model Without Disorder J. Phys. A 52 (2019) no.47, 474002

[13] N. Delporte and V. Rivasseau, The Tensor Track V: Holographic Tensors, PoS, (CORFU2017)218

[14] I. R. Klebanov, F. Popov, and G. Tarnopolsky. TASI Lectures on Large $N$ Tensor Models. PoS, TASI2017:004, 2018.

[15] R. Gurau. Universality for Random Tensors. Ann. Inst. H. Poincare Probab. Statist., 50(4):1474-1525, 2014. 
[16] R. Gurau V. Bonzom and M. Smerlak. Universality in $p$-spin glasses with correlated disorder. $J$. Stat. Mech., (L02003), 2013.

[17] T. Krajewski, M. Laudonio, R. Pascalie and A. Tanasa, Non-Gaussian disorder average in the Sachdev-Ye-Kitaev model,' Phys. Rev. D 99 (2019) no.12, 126014

[18] D J. Gross and V. Rosenhaus. A Generalization of Sachdev-Ye-Kitaev. JHEP, 1702:093, 2017.

[19] D. Stanford and E. Witten, Fermionic Localization of the Schwarzian Theory, JHEP 10 (2017), 008

[20] A. Kitaev and S. J. Suh, The soft mode in the Sachdev-Ye-Kitaev model and its gravity dual, JHEP 05 (2018), 183

[21] I. Aref'eva, M. Khramtsov, M. Tikhanovskaya and I. Volovich, Replica-nondiagonal solutions in the SYK model, JHEP 07 (2019), 113

[22] S. Dartois, V. Rivasseau and A. Tanasa, The $1 / N$ expansion of multi-orientable random tensor models, Annales Henri Poincare 15 (2014), 965-984

[23] S. Carrozza and A. Tanasa. $O(N)$ Random Tensor Models. Lett. Math. Phys., 106(11):1531-1559, 2016.

[24] D. Benedetti, S. Carrozza, R. Gurau and M. Kolanowski, The $1 / N$ expansion of the symmetric traceless and the antisymmetric tensor models in rank three," Commun. Math. Phys. 371 (2019) no.1, 55-97

[25] V. Bonzom, Tensor models with generalized melonic interactions arXiv:1905.01903 [math-ph]

[26] T. Krajewski and . Toriumi. Polchinski's exact renormalisation group for tensorial theories: Gaussian universality and power counting. J. Phys., A49(38):385401, 2016.

[27] J. Polchinski. Renormalization and Effective Lagrangians. Nucl. Phys., B231:269-295, 1984.

[28] Y. Gu, X. Qi and D. Stanford, Local criticality, diffusion and chaos in generalized Sachdev-Ye-Kitaev models, JHEP 05 (2017), 125 